\title{
The corona and companion of CoRoT-2a. Insights from X-rays and optical spectroscopy ${ }^{\star}$
}

\author{
S. Schröter, S. Czesla, U. Wolter, H. M. Müller, K. F. Huber, and J. H. M. M. Schmitt
}

\begin{abstract}
Hamburger Sternwarte, Universität Hamburg, Gojenbergsweg 112, 21029 Hamburg, Germany
e-mail: sschroeter@hs.uni-hamburg.de
\end{abstract}

Received 25 March 2011 / Accepted 23 May 2011

\begin{abstract}
CoRoT-2 is one of the most unusual planetary systems known to date. Its host star is exceptionally active, showing a pronounced, regular pattern of optical variability caused by magnetic activity. The transiting hot Jupiter, CoRoT-2b, shows one of the largest known radius anomalies. We analyze the properties and activity of CoRoT-2a in the optical and X-ray regime by means of a highquality UVES spectrum and a 15 ks Chandra exposure both obtained during planetary transits. The UVES data are analyzed using various complementary methods of high-resolution stellar spectroscopy. We characterize the photosphere of the host star by deriving accurate stellar parameters such as effective temperature, surface gravity, and abundances. Signatures of stellar activity, Li abundance, and interstellar absorption are investigated to provide constraints on the age and distance of CoRoT-2. Furthermore, our UVES data confirm the presence of a late-type stellar companion to CoRoT-2a that is gravitationally bound to the system. The Chandra data provide a clear detection of coronal X-ray emission from CoRoT-2a, for which we obtain an X-ray luminosity of $1.9 \times 10^{29} \mathrm{erg} \mathrm{s}^{-1}$. The potential stellar companion remains undetected in X-rays. Our results indicate that the distance to the CoRoT- 2 system is $\approx 270 \mathrm{pc}$, and the most likely age lies between 100 and $300 \mathrm{Ma}$. Our X-ray observations show that the planet is immersed in an intense field of high-energy radiation. Surprisingly, CoRoT-2a's likely coeval stellar companion, which we find to be of late-K spectral type, remains $\mathrm{X}$-ray dark. Yet, as a potential third body in the system, the companion could account for CoRoT-2b's slightly eccentric orbit.
\end{abstract}

Key words. stars: individual: CoRoT-2a - stars: fundamental parameters - planetary systems - stars: late-type - X-rays: stars

\section{Introduction}

The CoRoT-2 system stands out of the plethora of known exoplanet systems both for its exceptionally active host star and its unusually inflated planet. The hot Jupiter CoRoT-2b is the second transiting planet discovered by the space-based CoRoT mission (Alonso et al. 2008); its planetary nature was confirmed by spectroscopic follow-up observations with SOPHIE and HARPS (Bouchy et al. 2008). The planet orbits its host star every 1.74 days. Given its mass of $3.31 M_{\mathrm{J}}$ and large radius of $1.465 R_{\mathrm{J}}$ (Alonso et al. 2008), CoRoT-2b appears to be anomalously inflated in comparison to current evolutionary models (Guillot \& Havel 2011). A spectral analysis showed that its host star, CoRoT-2a, is a G7 dwarf with solar composition. Its spectrum shows strong Li I absorption and emission-line cores in $\mathrm{Ca}$ II $\mathrm{H}$ and $\mathrm{K}$, indicating that CoRoT-2a is a young and active star (Bouchy et al. 2008). CoRoT-2a has a close visual companion, 2MASS J19270636+0122577, separated by about $4^{\prime \prime}$. Photometric magnitudes from the optical to the infrared concordantly suggest that this object is a late-K or early-M type star located at the same distance as CoRoT-2 (Alonso et al. 2008; Gillon et al. 2010). Thus, CoRoT-2a and its visual companion possibly form a physical pair.

The continuous photometric data of CoRoT-2a provided by the CoRoT telescope span 152 days. CoRoT-2a's light curve shows a distinct pattern of variability caused by starspots. In several studies, the light curve was used to reconstruct the surface

* Based on observations obtained with UVES at the ESO VLT Kueyen telescope (program ID 385.D-0426) and the Chandra X-ray Observatory (obs. ID 10989). brightness distribution of CoRoT-2a: Lanza et al. (2009) applied a light-curve inversion technique and found that most spots are concentrated in two active longitudes of alternating strength located on opposite hemispheres. Moreover, it was demonstrated that starspots influence the profiles of transit light-curves and that this effect cannot be neglected in transit modeling (Wolter et al. 2009; Czesla et al. 2009). Because the latitudinal band eclipsed by the planet is accurately known (Bouchy et al. 2008), it is even feasible to study the spot coverage on the surface section recurrently eclipsed by the planet (Huber et al. 2009; Silva-Valio et al. 2010; Huber et al. 2010).

Secondary eclipses of CoRoT-2b were observed in the optical with CoRoT (Alonso et al. 2009; Snellen et al. 2010), in the infrared with Spitzer (Gillon et al. 2010; Deming et al. 2011), and from the ground (Alonso et al. 2010). While atmospheric models (Fortney et al. 2008) suggest the presence of a stratospheric thermal-inversion layer in CoRoT-2b caused by the strong irradiation, the observational situation remains inconclusive (e.g., Deming et al. 2011). The observed emission of the planet is currently incompatible with any kind of standard atmosphere model, and more sophisticated approaches including, for example, substantial carbon monoxide mass loss or additional substructure in the atmosphere may be needed to explain the observations (see Deming et al. 2011, for a discussion); the substantial activity of the host star adds another complicating factor to the picture (e.g., Knutson et al. 2010).

Using KECK/HIRES data, Knutson et al. (2010) searched for a relation between stellar activity as manifested by chromospheric emission in the $\mathrm{Ca}$ II $\mathrm{H}$ and $\mathrm{K}$ line cores and the emission spectra of hot Jovians. Among their sample of planet host-stars, 
CoRoT-2a stands out as being the most active as measured by its $\log R_{\mathrm{HK}}^{\prime}$ index. Ammler-von Eiff et al. (2009) reanalyzed the archival UVES data presented in Bouchy et al. (2008) (program 080.C-0661D) and determined precise estimates of CoRoT-2a's spectral properties such as effective temperature and iron abundance. In August 2009, Gillon et al. (2010) obtained and analyzed a new UVES spectrum (program 083.C-0174C). The authors provide a more detailed discussion of the $\mathrm{Li}$ absorption line and derive an age between 30 and $316 \mathrm{Ma}$ for CoRoT-2a. Additionally, they find evidence for a slight eccentricity of $0.014 \pm 0.008$ for the planetary orbit, which they attribute to the youth of the system, if not caused by CoRoT-2a's potential stellar companion.

Guillot \& Havel (2011) investigated the matter of CoRoT-2a's anomalously inflated planet on theoretical grounds by simultaneously modeling the planetary and stellar evolution including stellar activity. The authors' models favor two classes of solutions: either a young system with a star on the pre-main sequence (30-40 Ma) or a much older system ( $>100 \mathrm{Ma})$ with a main-sequence star. The authors discuss several effects that could have led to the anomalously large radius of CoRoT-2b. While they argue that the influence of starspots is minor, either the presence of additional infrared opacity sources in the planetary atmosphere that reduces the rate of heat loss during the planet's evolution or a recent interaction with a third body in the system that leaves the planet in an eccentric orbit could account for the observed radius anomaly.

In this work, we present a new UVES spectrum of CoRoT-2a and its visual companion, which we analyzed in detail to refine the spectroscopic parameters and chromospheric activity indicators. We especially aim at deriving several independent estimates of the age and the distance of the CoRoT-2 system. First, we present the results of our analysis of the high-resolution UVES spectrum of CoRoT-2a and our low-quality spectrum of its visual companion. Second, we present our analysis of the first X-ray observation of CoRoT-2a. We proceed by discussing the physical implications of our findings (Sect. 4) and, finally, we present our conclusions.

\section{Optical spectroscopy with UVES}

\subsection{Observations}

On June 7, 2010, we acquired 24 high-resolution spectra of CoRoT-2a with the UVES spectrograph (Dekker et al. 2000) mounted at the VLT Kueyen telescope (program ID 385.D-0426(A)). The instrument was set up in "Dichroic 2" mode with a slit width of 0.7 ". We used the crossdisperser \#4 with a central wavelength of $7600 \AA$ providing a wavelength coverage of 3800-5000 $\AA$ on the blue arm and 5700-9500 ̊ on the red arm. Around $7600 \AA$ a section of $100 \AA$ is missing because of the gap between the two detectors. Because no overbinning was applied during CCD read-out, we reach a spectral resolving power of about 60000 .

In this set-up, we obtained 24 individual spectra with exposure times of $800 \mathrm{~s}$ each. The observations were scheduled to cover a full planetary transit of CoRoT-2b including a reasonable time span before and after the actual transit event. Owing to worsening seeing conditions, the last 13 observations were carried out using the image slicer to reduce light losses. Additionally, we used an archived UVES spectrum obtained on Oct. 13, 2007 in the framework of the program 080.C-0661(D) for comparison.
To reduce the UVES echelle data, we applied the UVES pipeline in version 4.7.8 with its associated standard recipes and the REDUCE package developed by Piskunov \& Valenti (2002). Our analysis is based on the REDUCE spectra unless stated otherwise. Background-sky subtraction for exposures taken with the image slicer is difficult because there is hardly any sky area left on the detector that could be used to extract the background. Indeed, the UVES pipeline does currently not apply any such subtraction for exposures taken with the image slicer. The 24 individual UVES spectra were combined and yielded a highresolution spectrum of CoRoT-2a with a signal-to-noise ratio (SNR) of about 200 at $6500 \AA$. Although sky emission-lines are present in the spectrum, they did not affect our analysis.

\subsection{Analysis of optical data}

The stellar spectrum conveys information about the physical conditions in the stellar atmosphere. The parameters of primary interest in our analysis were the effective temperature, elemental abundances, surface gravity, and microturbulence velocity. Unfortunately, those parameters cannot be determined independently from the observed stellar spectrum, but they are highly correlated. Several techniques are commonly used in spectroscopic analyses, and the results depend on the underlying assumptions and implementation. Systematic errors can originate in the data reduction process or in differences in the adopted atomic data. As a consequence, error bars based on purely statistical considerations usually underestimate the true uncertainty. Therefore, we applied a number of independent analyses to corroborate the validity of our parameter and error estimates. In the following analysis, we concentrate on the time-averaged UVES spectrum of CoRoT-2a. An analysis of the temporally resolved properties will be presented in another context.

\subsection{Elemental abundances via excitation/ionization balance}

In our high-resolution UVES spectrum of CoRoT-2a, we measured equivalent widths (EWs) of lines of neutral (Fe I) and ionized iron (Fe II) and several other metals (Na, Mg, Al, Si, Ca, $\mathrm{Ti}, \mathrm{V}, \mathrm{Cr}, \mathrm{Mn}, \mathrm{Co}, \mathrm{Ni}$, and $\mathrm{Ba})$. Our selection of lines is based on the line lists provided by Sousa et al. (2008) and Bubar \& King (2010), out of which we compiled a list of iron lines without severe blends with excitation potentials below $5 \mathrm{eV}$ and EWs between 10 and $200 \mathrm{~m} \AA$. Our resulting line list comprises $212 \mathrm{Fe}_{\mathrm{I}}$ lines, $26 \mathrm{Fe}$ II lines, and 162 lines of other metals.

To measure the EWs of a large number of spectral lines, Sousa et al. (2007) developed the ARES ${ }^{1}$ code. This algorithm detects spectral lines by evaluating numerical derivatives of the spectrum. For the following analysis, we set up our own implementation of the ARES algorithm, extending it at several points, for example, by a low-pass Fourier filter to suppress noise effects and an estimation of the local continuum. Our tool runs semiautomatically, allowing the user to interactively improve the fit result by visual inspection where desired. The normalization of the spectrum is made manually by comparing the observed to a synthetic spectrum to identify regions of undisturbed continuum, which are then used as nodes for a linear (or cubic) spline fit.

The EWs thus obtained were used as input for the 2010 version of $\mathrm{MOOG}^{2}$ (Sneden 1973) together with ATLAS planeparallel model atmospheres ${ }^{3}$ (Kurucz 1993). We used MOOG

\footnotetext{
1 See http://www.astro.up.pt/ sousasag/ares/

2 See http://www.as.utexas.edu/ chris/moog.html

${ }^{3}$ See http://kurucz.harvard.edu/grids.html
} 
Table 1. Stellar parameters and iron abundance of CoRoT-2a determined from the analysis of Fe I and II lines, the Sousa et al. (2007) line-ratio technique, and SME line profile fitting.

\begin{tabular}{lcccccc}
\hline \hline Stellar parameters & $T_{\text {eff }}[\mathrm{K}]$ & $\log g(\mathrm{cgs})$ & $\xi_{t}\left[\mathrm{~km} \mathrm{~s}^{-1}\right]$ & {$[\mathrm{Fe} / \mathrm{H}]$} & $N(\mathrm{Fe} \mathrm{I}, \mathrm{Fe}$ II) & Source \\
\hline Excitation/ionization balance & $5598 \pm 34$ & $4.47 \pm 0.14$ & $1.75 \pm 0.04$ & $0.04 \pm 0.02$ & 212,26 & this work (Sect. 2.3) \\
Excitation/ionization balance & $5608 \pm 37$ & $4.71 \pm 0.20$ & $1.49 \pm 0.06$ & $0.07 \pm 0.04$ & 26,9 & Ammler-von Eiff et al. (2009) \\
Fe I and II lines & $5625 \pm 120$ & $4.3 \pm 0.2$ & $0.0 \pm 0.1$ & & Bouchy et al. (2008) \\
Line ratio calibration & $5513 \pm 111$ & & & & this work (Sect. 2.4) \\
SME global fit & $5475 \pm 44$ & $4.62 \pm 0.06$ & 1.52 & $-0.06 \pm 0.03$ & this work (Sect. 2.5) \\
H $\alpha$ t6563 & $5510_{-70}^{+90}$ & & & & this work (Sect. 2.5) \\
H $\alpha 6563$ & $5450 \pm 120$ & & & & Bouchy et al. (2008) \\
Ca I $\lambda 6122, \lambda 6162, \lambda 6439$ & & $4.49 \pm 0.14$ & & this work (Sect. 2.5) \\
Na D $\lambda 5890, \lambda 5896$ & & $4.53 \pm 0.18$ & & & this work (Sect. 2.5) \\
\hline
\end{tabular}

to derive the effective temperature by minimizing the correlation between iron abundance and excitation potential, whereas the microturbulence velocity was obtained by removing the correlation with reduced EW, i.e., the EW normalized by the central wavelength of the line. The surface gravity is derived by minimizing the difference between the resulting $\mathrm{Fe}_{\mathrm{I}}$ and Fe II abundances.

To account for possible errors in the atomic line parameters, the spectroscopic analysis proceeds differentially to the Sun. Therefore, we measured each EW in both a lunar UVES spectrum provided by the ESO Quality Control and Data Processing Group ${ }^{4}$ and the stellar spectrum and subsequently subtracted the solar abundance from the resulting stellar abundance. We used the freely available PYSPEC ${ }^{5}$ Python interface to MOOG by Bubar for our differential spectroscopic analysis (for details, see Bubar \& King 2010). The abundances of the other metals were derived in the same way, but keeping the input stellar atmosphere model fixed.

We present our results in Table 1. The listed errors on effective temperature and microturbulent velocity are estimated by investigating the correlation of the iron abundance with excitation potential and reduced EW, respectively, as measured by Spearman's rank correlation coefficient. In particular, the parameters were varied until a $1 \sigma$ correlation was found, and the associated values were then used as an estimator of the reasonable parameter range. The error on $\log g$ was propagated based on the errors of the remaining parameters as detailed in Bubar \& King (2010). Our spectral parameters agree well with earlier results obtained by Bouchy et al. (2008) and Ammler-von Eiff et al. (2009) from the analysis of Fe I and II lines.

The inferred elemental abundances are summarized in Table 2. Neither for Ni nor for Ti I and II did we find any strong correlations of abundance with excitation potential or EW given the atmospheric model derived from the Fe I and II lines. This indicates that the stellar parameters were indeed correctly chosen. Within the errors the elemental abundances are compatible with the solar values. The overabundance of $\mathrm{Ba}$ II is a result of the lines being blended with Fe lines. We therefore redetermined the Ba II abundance via line synthesis and obtained a value of $+0.13 \pm 0.09$ dex, which better agrees with the remaining elemental abundances.

\subsection{Effective temperature via line ratios}

Comparing spectral lines with different temperature sensitivity with each other provides a valuable temperature diagnostic.

\footnotetext{
${ }_{4}$ See http://www.eso.org/observing/dfo/quality/

5 See

http://www.pas.rochester. edu/ ebubar/speclink.html
}

Table 2. Elemental abundances for CoRoT-2a relative to the Sun with the number of lines $N(\mathrm{X})$ used for each element.

\begin{tabular}{lcc}
\hline \hline Elem. & {$[\mathrm{X} / \mathrm{H}]$} & $N(\mathrm{X})$ \\
\hline $\mathrm{Mg}_{\mathrm{I}}$ & $-0.16 \pm 0.13$ & 4 \\
$\mathrm{Si}_{\mathrm{I}}$ & $-0.05 \pm 0.11$ & 17 \\
$\mathrm{Ca}_{\mathrm{I}}$ & $+0.12 \pm 0.10$ & 14 \\
$\mathrm{Ti}_{\mathrm{I}}$ & $+0.05 \pm 0.14$ & 25 \\
$\mathrm{Ti}_{\text {II }}$ & $-0.02 \pm 0.15$ & 13 \\
$\mathrm{~V}_{\text {I }}$ & $+0.05 \pm 0.10$ & 12 \\
$\mathrm{Cr}_{\mathrm{I}}$ & $+0.03 \pm 0.27$ & 18 \\
$\mathrm{Cr}_{\text {II }}$ & $+0.05 \pm 0.16$ & 3 \\
\hline
\end{tabular}

\begin{tabular}{lcc}
\hline \hline Elem. & {$[\mathrm{X} / \mathrm{H}]$} & $N(\mathrm{X})$ \\
\hline $\mathrm{Mn}$ I & $+0.05 \pm 0.15$ & 5 \\
$\mathrm{Co}$ I & $-0.13 \pm 0.10$ & 4 \\
$\mathrm{Ni}$ I & $-0.10 \pm 0.10$ & 38 \\
$\mathrm{Na}$ I & $-0.03 \pm 0.04$ & 2 \\
$\mathrm{Al}$ I & $+0.01 \pm 0.10$ & 4 \\
$\mathrm{Ba} \mathrm{II}$ & $+0.25 \pm 0.05$ & 3 \\
$\mathrm{Ba}_{\text {II }}{ }^{a}$ & $+0.13 \pm 0.09$ & \\
$\mathrm{Li} \mathrm{I}^{b}$ & $+1.55 \pm 0.38$ & \\
\hline
\end{tabular}

Notes. ${ }^{(a)}$ See discussion in Sect. 2.3. ${ }^{(b)}$ See discussion in Sect. 2.6.

Particularly useful is comparing the EWs of spectral lines belonging to metallic species. A line-ratio technique based on such a comparison was proposed by Sousa et al. (2007), who also calibrated their method using 451 FGK dwarf stars (Sousa et al. 2010). The authors determine an empirical relation between effective temperature and the EW ratio for a set of 433 pairs of spectral lines and incorporated their results into the "Teff_LR Code" code, which is an extension to the ARES code; both are freely available.

We used the relations published in Sousa et al. (2007, 2010) and obtained a value of $T_{\text {eff }}=5513 \pm 111 \mathrm{~K}$ for CoRoT-2a's effective temperature, using 322 metallic lines and 22 independent line ratios. Although somewhat lower, this value is consistent with previous estimates (cf. Table 1). As a cross-check, we determined the solar effective temperature using the same set of lines in the UVES solar spectrum and found the resulting value of $5784 \pm 152 \mathrm{~K}$ to agree well with the literature (Cox 2000, p. 341).

\subsection{Synthetic spectra fitting via SME}

As an alternative to the modeling of line EWs, the stellar parameters can also be obtained by directly fitting the profile of spectral lines using synthetic spectra. This approach is implemented in the "Spectroscopy Made Easy" (SME) package (version 2.1; Valenti \& Piskunov 1996). This interpolates on a Kurucz grid of stellar atmospheres and employs a VALD 6 (Piskunov et al. 1995) line list to compute a synthesized spectrum for each set of stellar parameters. The observed spectrum is fitted by minimizing the residuals via a non-linear least-squares algorithm.

We used SME to determine the stellar parameters first in a global fit and second by fitting individual lines sensitive to $T_{\text {eff }}$ and $\log g$. Currently, it is not feasible to compute a reliable error

\footnotetext{
${ }^{6}$ See http://www. astro.uu. se/ vald/php/vald.php
} 
estimate for a global fit due to the large computational effort of calculating synthetic spectra. From the analysis of a set of 1040 FGK stars, however, Valenti \& Fischer (2005) derived typical errors of $44 \mathrm{~K}$ in $T_{\text {eff }}, 0.06 \mathrm{dex}$ in $\log g$, and 0.03 dex in metallicity, which we adopt below. For the analysis of single line profiles, we usually fixed all parameters at their best-fit values and obtained the error by computing the $90 \%$ confidence inter$\operatorname{val}\left(\Delta \chi^{2}=2.71\right)$.

An approximation of the effective temperature can be obtained by investigating the $\mathrm{H} \alpha$ line profile. The wings of the prominent $\mathrm{H} \alpha$ line at a nominal wavelength of $6563 \AA$ are sensitive to a wide range of effective temperatures of G- and F-type stars (e.g. Fuhrmann 2004), while remaining reasonably unaffected by the surface gravity, $\log g$, and the metallicity.

However, active late-type stars are known to show strong contributions of chromospheric emission in the Balmer lines, which can even extend into the wings of the line profiles (e.g., Montes et al. 1997). This can interfere with the determination of the effective temperature. We independently analyzed the line wings of $\mathrm{H} \alpha$ and $\mathrm{H} \beta$. Consistent results for the temperatures deduced from both Balmer lines indicate that the wings of $\mathrm{H} \alpha$ are not strongly affected by chromospheric activity (Fuhrmann 2004; König et al. 2005).

A visual inspection of the symmetry of the $\mathrm{H} \alpha$ line profile suggested that the UVES pipeline, in this respect, provided a superior result, so that we rely on the pipeline spectra during this analysis. We note, however, that in any case a manual rectification of the spectrum is necessary. Hence, this method is prone to considerable uncertainties. We used SME to fit synthetic spectra to the observed $\mathrm{H} \alpha$ and $\mathrm{H} \beta$ line profiles excluding the line cores and found an effective temperature $T_{\text {eff }}$ of $5510_{-70}^{+90} \mathrm{~K}$ and $5520_{-90}^{+80} \mathrm{~K}$, respectively. This is consistent with the result of $5450 \pm 120 \mathrm{~K}$ derived from the analysis of the $\mathrm{H} \alpha$ line observed with HARPS (Bouchy et al. 2008). The good agreement between the Balmer line estimates indicates that the chromospheric contribution remains small.

Several pressure-broadened spectral lines can be used to determine the surface gravity of late-type stars. Examples of these lines are the $\mathrm{Mg}$ Ib triplet (Fuhrmann 1998), the Na I D doublet, and the Ca I lines at 6122, 6162, and $6439 \AA$ (e.g., Bruntt et al. 2010). Because of the gap between the two detectors, the spectrum does not contain any $\mathrm{Mg}$ Ib lines. We therefore concentrated on the $\mathrm{Na}$ and $\mathrm{Ca}$ lines. With SME, we iteratively fitted synthetic spectra to the three $\mathrm{Ca}$ I lines and the $\mathrm{Na} \mathrm{D}$ line, leaving only $\log g$ as a free parameter. The resulting value from the Ca I lines $(4.49 \pm 0.14)$ was found to be consistent with the value derived from $\mathrm{Na} \mathrm{D}(4.53 \pm 0.18)$.

\subsection{The age of CoRoT-2a as determined by Li I}

The abundance of lithium is a valuable indicator of the stellar age. The element is depleted by lithium burning primarily during the early phases of stellar evolution, when the existence of deep convection zones allows for the interchange of material between the stellar interior and the surface (e.g., Pinsonneault 1994).

CoRoT-2a shows a strong Li line at $\approx 6708 \AA$, for which we determined an EW of $139 \pm 1 \mathrm{~m} \AA$. We used SME to fit synthetic spectra with all remaining stellar parameters kept fixed and derived an abundance of $A_{\mathrm{Li}}=+2.6 \pm 0.3$. This value confirms the result of Gillon et al. (2010), who found $A_{\mathrm{Li}}=+2.8$. According to Sestito \& Randich (2005), this Li content is typically found in G-type stars of $T_{\text {eff }}=5600 \mathrm{~K}$ at an age between 100 and $250 \mathrm{Ma}$.

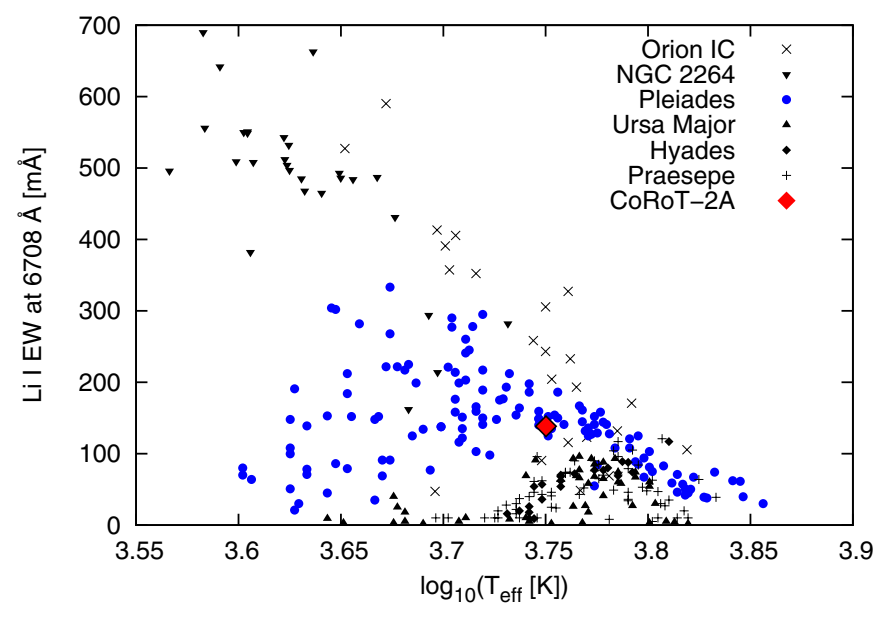

Fig. 1. Li I EW vs. effective temperature for CoRoT-2a and a sample of open stellar clusters with different ages.

In Fig. 1 we show effective temperature versus Li I line EW for the open stellar clusters Orion IC (10 Ma), NGC 2264 (10 Ma), Pleiades (100 Ma), Ursa Major (300 Ma), Hyades (660 Ma), and Praesepe (660 Ma) (King 1993; Soderblom et al. 1990, 1993a,b,c, 1999), additionally, the location of CoRoT-2a is marked in the diagram. The clusters are of different age, so that putting CoRoT-2a in the context of the cluster properties provides an indication of its age. The Li I EW of CoRoT-2a is best compatible with those in the Pleiades, indicating an age of about $100 \mathrm{Ma}$. This finding is consistent with the numbers derived by Guillot \& Havel (2011) from evolutionary modeling and also the age estimates given by Gillon et al. (2010), who derive an age between 30 and $316 \mathrm{Ma}$.

\subsection{Activity indicators}

Active stars are known to show strong emission in the $\mathrm{Ca}$ II $\mathrm{H}$ and $\mathrm{K}$ line cores at 3934.8 and $3969.7 \AA$ (see Linsky 1980, for a profound discussion). This is also true for CoRoT-2a; we show its $\mathrm{Ca}$ II $\mathrm{H}$ and $\mathrm{K}$ lines in Fig. 2.

In late-type stars the width, $W_{0}$, of the emission cores seen in the $\mathrm{Ca}$ II $\mathrm{H}$ and $\mathrm{K}$ lines is mainly sensitive to the value of $\log g$, and, hence, to the mass and radius of the star, but insensitive to the effective temperature and metallicity. If calibrated appropriately, the width can be used as a rough estimator of the absolute luminosity of a star (Wilson \& Bappu 1957). We used the recent calibration of Pace et al. (2003) together with CoRoT-2a's apparent magnitude corrected for interstellar extinction (see Sect. 4.3) to obtain a distance estimate for the CoRoT-2 system. Pace et al. find no significant effect of rotational and instrumental broadening within their sample, which comprises stars with $v \sin i<14 \mathrm{~km} \mathrm{~s}^{-1}$. Neglecting broadening effects, we obtained a distance estimate of $190_{+60}^{-50} \mathrm{pc}$. When both broadening mechanisms were taken into account by a quadratic correction to $W_{0}$ as described by Pace et al., the distance estimate reduced to $140_{-40}^{+50} \mathrm{pc}$, which is lower but still compatible, given the errors.

The Wilson chromospheric flux index $\left(S_{\mathrm{MW}}\right.$; Vaughan et al. 1978; Baliunas et al. 1998) is a popular measure of chromospheric activity. To estimate $S_{\mathrm{MW}}$ from our spectra, we used the calibration procedure for UVES spectra described by Melo et al. (2006). These authors defined a proper index $S_{\text {US }}$ and 


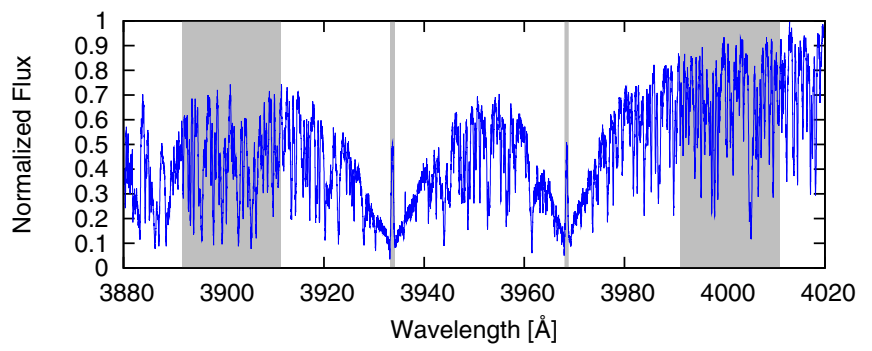

Fig. 2. Region of CoRoT-2a's spectrum containing the Ca II $\mathrm{H}$ and $\mathrm{K}$ lines. Gray shades indicate the bands defined by Melo et al. (2006) for the definition of $\log R_{\mathrm{HK}}^{\prime}$. See text for details.

determined the following relation between their index and the Wilson index:

$S_{\mathrm{US}}=0.06111 \times S_{\mathrm{MW}}-0.00341$

(see Eq. (1) in Melo et al. 2006). Inverting this relation, we obtained an $S_{\text {MW }}$ index of 0.479 . We proceeded by correcting our $S_{\text {MW }}$ index for the color dependence (Rutten 1984) and subtracted the expected photospheric contribution to the flux in the line cores (Noyes et al. 1984). Using a value of 0.854 for the $B-$ $V$ color of CoRoT-2a from the SIMBAD database ${ }^{7}$, we obtained an emission ratio of $\log R_{\mathrm{HK}}^{\prime}=-4.458 \pm 0.051$, which agrees well with the results of Gillon et al. (2010) $(-4.471 \pm 0.0629)$ and Knutson et al. (2010) (-4.331).

The central part of the $\mathrm{Ca}$ II $\mathrm{H}$ and $\mathrm{K}$ emission core does not show the double-horned structure usually observed in the line cores of solar-like stars. We found no sign of self-reversal in the central part of the line core. According to Ayres (1979), strong chromospheric heating through stellar activity leads to a decrease of the wavelength separation between the two $\mathrm{K}_{2}$ peaks of the emission core, so that the central $\mathrm{K}_{3}$ dip is easily obliterated by instrumental, macroscopic, and microscopic broadening effects. The lack of a detectable self-reversal is, therefore, an indicator of strong chromospheric heating itself. Because chromospheric heating causes an increase in the wavelength separation of the two $\mathrm{K}_{1}$ minima that approximately counterbalances the mutual approach of the $\mathrm{K}_{2}$ peaks, the Wilson-Bappu width, $W_{0}$, remains basically insensitive to stellar activity.

Chromospheric activity can also be measured in the $\mathrm{Ca}$ II infrared triplet (IRT) at 8498, 8542, and $8662 \AA$ (see e.g., Andretta et al. 2005; Busà et al. 2007). To correctly assess the chromospheric contribution to the line profile, the line forming process within the photosphere must be accurately modeled. Andretta et al. (2005) proposed the activity indicator $R_{\mathrm{IRT}}$ defined as the difference between the central line depths (or central depressions) of the observed spectrum and a rotationally-broadened NLTE model. We calculated the central depression, $R_{\mathrm{IRT}}$, and $\Delta W_{\mathrm{IRT}}$, i.e., the EW of the residual line profile and the corresponding error estimates following the approach detailed in Busà et al. (2007). In our calculations, we used LTE models synthesized via SME, which, according to Andretta et al., suffice to approximate the photospheric spectrum for main-sequence stars of solar metallicity. The parameters thus obtained are summarized in Table 3. The CoRoT-2a Ca IRT along with our synthetic templates are shown in Fig. 3, which also indicates the residuals. Busà et al. found a relationship between the $\log R_{\mathrm{HK}}^{\prime}$ activity index and $R_{\mathrm{IRT}}$, however, with a large scatter. Given our value of $\log R_{\mathrm{HK}}^{\prime}=4.46$, the relation predicts a value of 0.25 for $R_{\mathrm{IRT}}$, which reasonably agrees with our results.

\footnotetext{
7 See http://simbad.u-strasbg.fr/simbad/
}

Table 3. Central depression $(C D), R_{\mathrm{IRT}}$ and residual equivalent width $\Delta W_{\text {IRT }}$ for the lines of the Ca II infrared triplet in CoRoT-2a.

\begin{tabular}{cccc}
\hline \hline$\lambda[\AA]$ & $C D_{\text {obs }}$ & $R_{\text {IRT }}$ & $\Delta W_{\text {IRT }}[\mathrm{m} \AA]$ \\
\hline 8498 & $0.266 \pm 0.009$ & $0.342 \pm 0.009$ & $237.6 \pm 1.6$ \\
8542 & $0.361 \pm 0.006$ & $0.297 \pm 0.006$ & $63.1 \pm 0.6$ \\
8662 & $0.413 \pm 0.014$ & $0.256 \pm 0.014$ & $171.4 \pm 2.1$ \\
\hline
\end{tabular}

\subsection{Interstellar absorption features}

Analyzing the emission line cores of the $\mathrm{Ca}$ II $\mathrm{H}$ and $\mathrm{K}$ complex, we found narrow $\mathrm{K}_{4}$ (see e.g., Reimers 1977, for designation) and $\mathrm{H}_{4}$ absorption features close to both central chromospheric emission peaks (Fig. 4). We therefore attribute the features to $\mathrm{Ca}$ II absorption and determine a barycentric velocity of $-15 \mathrm{~km} \mathrm{~s}^{-1}$, corresponding to a relative velocity of about $+40 \mathrm{~km} \mathrm{~s}^{-1}$ with respect to CoRoT-2a.

Separately considering our 24 UVES spectra observed within $6 \mathrm{~h}$ and the archived UVES spectrum taken about three years earlier in 2007, we found no intrinsic variability of this feature. The EW of the $\mathrm{Ca}$ II $\mathrm{K}_{4}$ absorption feature is $65 \mathrm{~m} \AA$,

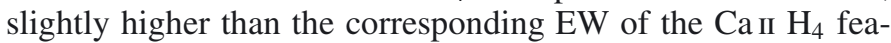
ture with $53 \mathrm{~m} \AA$. These blue shifted absorption features of ionized material may be caused by wind absorption as observed in giant stars (e.g., Reimers 1977) or, alternatively, by the interstellar medium.

While $\mathrm{Ca} \mathrm{H}_{4}$ and $\mathrm{K}_{4}$ features caused by wind absorption are observed in giant stars rather than dwarfs such as CoRoT-2a, the narrowness of the features is surprising if they are attributed to interstellar absorption. Often, multiple interstellar clouds are found in the line of sight, giving rise to a more diffuse absorption feature. Indeed, the width of the $\mathrm{Ca} \mathrm{H}_{4}$ and $\mathrm{K}_{4}$ features is comparable to that of telluric lines in our spectrum. However, considering the temporal stability, we argue in favor of interstellar absorption as the origin of the $\mathrm{Ca}_{4}$ and $\mathrm{K}_{4}$ features.

The Sun is situated within a region pervaded by hot, ionized plasma of low density known as the Local Cavity that reaches a radius of about 100 pc (e.g., Cox \& Reynolds 1987; Sfeir et al. 1999). This cavity contains many diffuse clouds with a complex velocity structure, which typically give rise to $\mathrm{Ca}$ II absorption features with EWs of a few mA (Lallement \& Bertin 1992). The density distribution of $\mathrm{Ca}$ II is fairly uniform in the interstellar medium beyond the Local Cavity, so that the EW of interstellar $\mathrm{Ca}$ II $\mathrm{H}$ and $\mathrm{K}$ absorption can be used as a distance indicator for sufficiently distant objects (Megier et al. 2005, 2009; Welsh et al. 2010). Using Eqs. (1) and (2) in Megier et al. (2005), we translated the EWs of both Ca II absorption features into distance estimates of $274 \mathrm{pc}$ for the $\mathrm{Ca}_{\mathrm{II}} \mathrm{K}_{4}$ feature and $256 \mathrm{pc}$ for the $\mathrm{Ca}$ II $\mathrm{H}_{4}$ feature.

We note that our spectrum of CoRoT-2a also shows two strong, nearly saturated $\mathrm{Na}$ I absorption lines with the same radial velocity shift within the blue wing of the $\mathrm{Na}$ I $\mathrm{D}$ doublet. These broad absorption features have EWs of $270 \mathrm{~m} \AA\left(\mathrm{D}_{2}\right)$ and $230 \mathrm{~m} \AA\left(\mathrm{D}_{1}\right)$, which is again compatible with interstellar absorption and a distance of $340 \pm 180 \mathrm{pc}$ and $320 \pm 140 \mathrm{pc}$, respectively (Welsh et al. 2010). Additional interstellar absorption lines of $\mathrm{K}_{\mathrm{I}}$ at $7698.974 \AA$ or molecules such as $\mathrm{CH}$ could not be detected.

In summary, we estimate a distance of $270 \pm 120 \mathrm{pc}$ for CoRoT-2a, based on interstellar absorption; the error was estimated from the standard deviation of the distances of the stars with $\mathrm{K}_{4}$ EWs in a $\pm 5 \mathrm{~m} \AA$ band around our measured EW (cf., Fig. 5). 


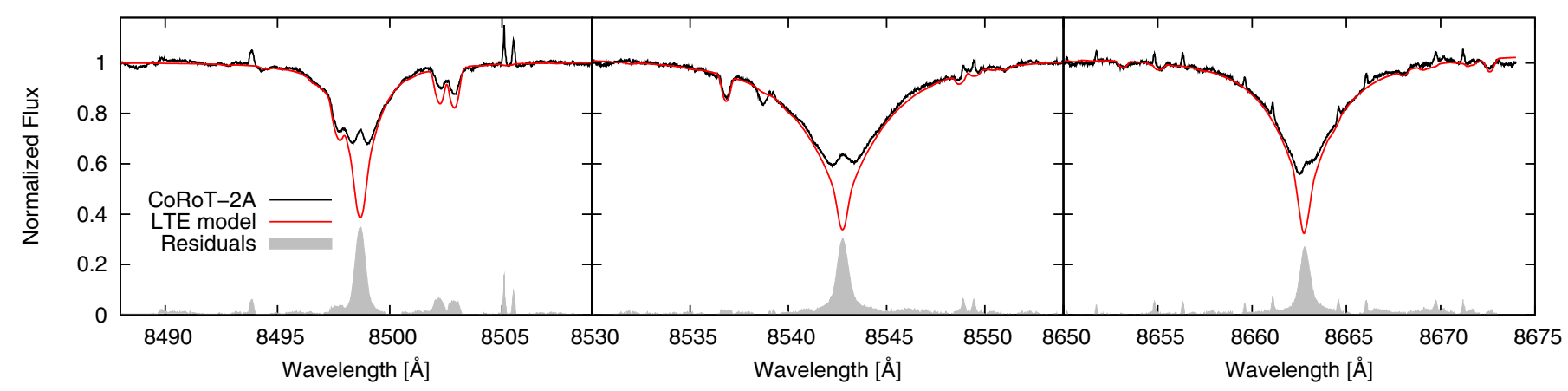

Fig. 3. Ca II infrared triplet in the spectrum of CoRoT-2a (black), which is used as activity diagnostic. The activity indicators are calculated relative to an LTE synthetic spectrum (red) based on the residuals (gray) as outlined in Busà et al. (2007). The small emission features result from sky emission (cf., Sect. 2.1).

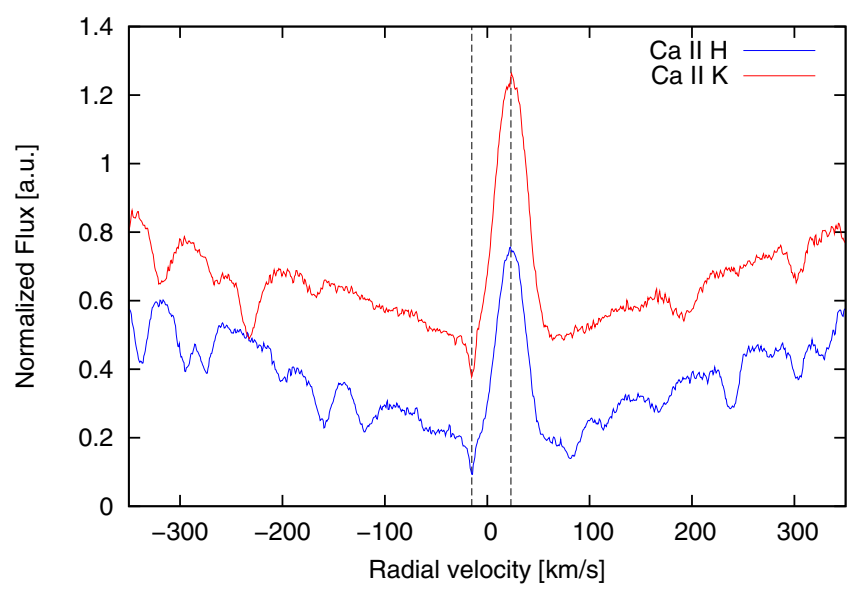

Fig. 4. Emission line cores of the $\mathrm{Ca}$ II $\mathrm{H}$ and $\mathrm{K}$ lines clearly showing the $\mathrm{H}_{4}$ and $\mathrm{K}_{4}$ absorption features. The spectrum of CoRoT-2a is shifted in radial velocity by $23.2 \mathrm{~km} \mathrm{~s}^{-1}$, while the absorption features show a barycentric velocity shift of $-15 \mathrm{~km} \mathrm{~s}^{-1}$.

\subsection{Evidence for a gravitationally bound companion}

CoRoT-2a has a close neighbor, 2MASS J19270636+0122577, about $4 "$ in southeast direction. Alonso et al. (2008) found that the color of this visual companion is consistent with a late-K or early-M type star located at the same distance as CoRoT-2a. During five of our 24 UVES observations this nearby neighbor was placed inside the slit along with CoRoT-2a, which we used to obtain a low SNR spectrum of the companion. The separation of the two objects allowed us to separately extract the spectra using the UVES pipeline.

Because the companion is $\approx 3.5$ mag fainter than CoRoT-2a in the visual band, the resulting spectrum has an SNR of no more than 10-20 depending on wavelength. It is dominated by absorption lines from neutral and singly-ionized metals. In particular, we find strong absorption in $\mathrm{Ca} I$ and $\mathrm{Mg}$ II, whereas the Ca II lines are comparably weak. Furthermore, we find a relatively weak $\mathrm{H} \alpha$ line and distinct edges caused by titanium oxide (TiO) absorption.

The TiO bands are a valuable indicator of stellar effective temperature if the metallicity is known, while they are less sensitive to surface gravity (Milone \& Barbuy 1994). To obtain an estimate of the effective temperature, we compared the coadded companion spectrum to synthetic spectra calculated with

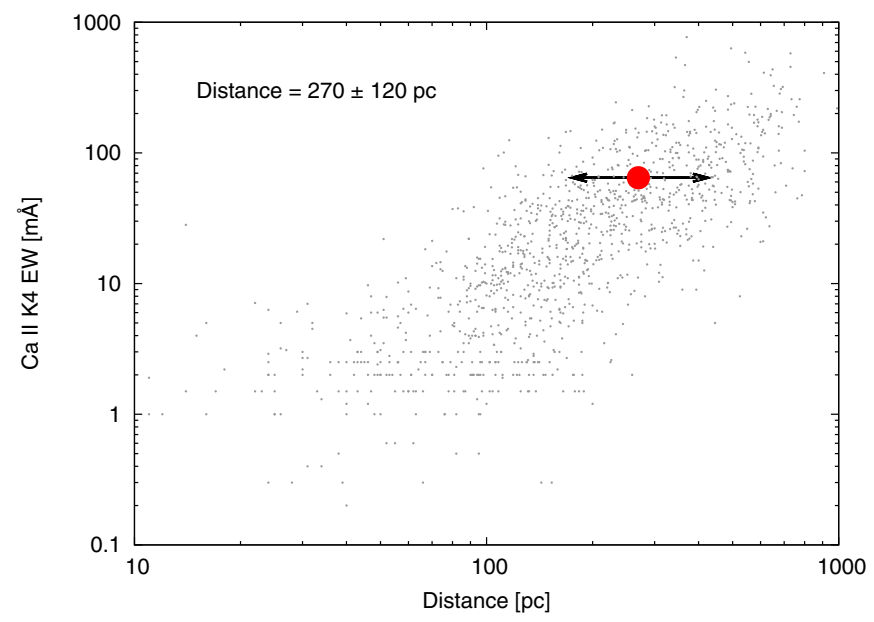

Fig. 5. Equivalent width of the interstellar $\mathrm{Ca}$ II $\mathrm{K}_{4}$ absorption line versus distance for stars within $800 \mathrm{pc}$ (catalog data compiled by Welsh et al. 2010). The measured Ca II $\mathrm{K}_{4}$ absorption of CoRoT-2a is consistent with a distance of roughly $300 \mathrm{pc}$.

SPECTRUM $^{8}$ (Gray \& Corbally 1994) using line lists containing the $\mathrm{TiO}$ and $\mathrm{ZrO}$ lines compiled by Plez (1998) ${ }^{9}$.

Assuming solar metallicity, we set up a Markov-Chain Monte-Carlo (MCMC) framework to find an estimate of the effective temperature and the associated error. We used uniform priors on the stellar parameters and allowed for an additional normalization constant accounting for inadequacies during blaze correction and continuum normalization. We focused on the strongest $\mathrm{TiO}$ band with its bandhead at $7054 \AA$ and analyzed the three absorption edges individually. The resulting three $95 \%$ credibility intervals for $T_{\text {eff }}$ consistently yield an effective temperature between 3900 and $4100 \mathrm{~K}$ (see Fig. 6). The surface gravity was found to be $\log g=4.9 \pm 0.1$, which is very close to our imposed upper bound of $\log g=5$ and does not seem to be well constrained.

We tried to use SME to fit synthetic spectra to several spectral lines known to be sensitive to the stellar parameters. Our efforts were, however, strongly hampered by the low SNR of the spectral data owing to the faintness of the companion. From the analysis of the $\mathrm{Na}$ I doublet at $5890 \AA$, the $\mathrm{Ca}$ I lines at 6122 , 6162 , and $6439 \AA$, and a set of 137 single Fe lines, we found the stellar spectrum to be best described by effective temperatures

\footnotetext{
8 See http://www1.appstate.edu/dept/physics/spectrum

9 See http://www.graal. univ-montp2.fr/hosted/plez
} 

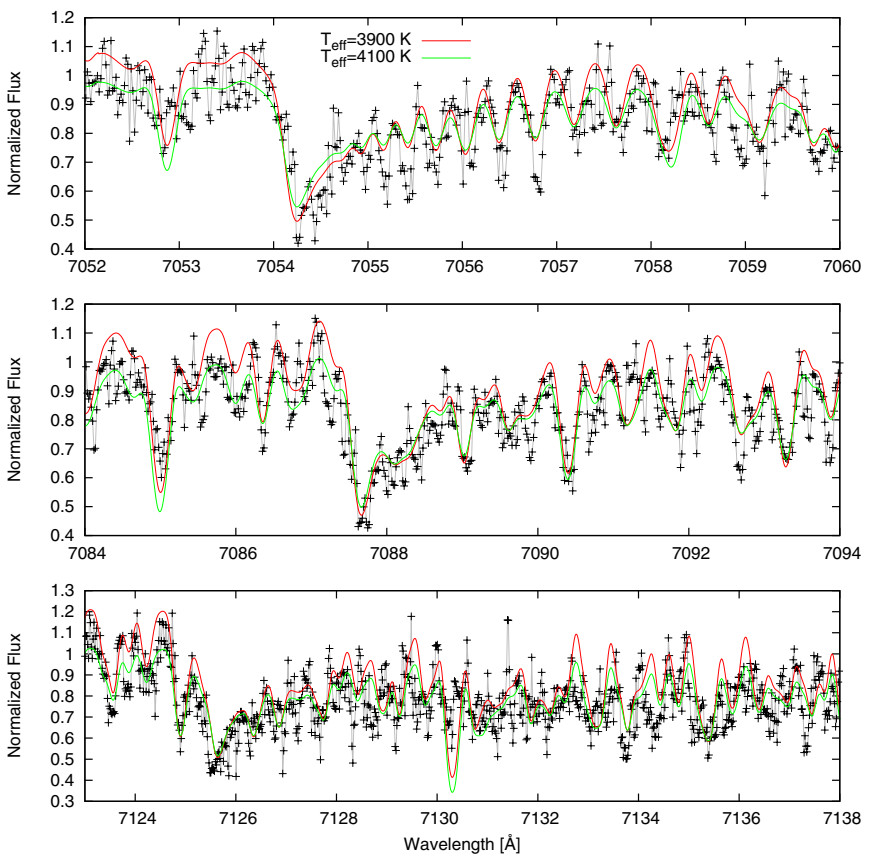

Fig. 6. Part of the companion spectrum showing the temperaturesensitive TiO band at 7050-7200 $\AA$. The red/green lines are synthetic spectra for $T_{\text {eff }}=3900 \mathrm{~K}$ and $4100 \mathrm{~K}$, respectively, computed for solar metallicity and $\log g=4.9$.

around $4000 \mathrm{~K}$ and a surface gravity between 4.6 and 4.9. Unfortunately, the quality of the spectrum made an analysis of the $\mathrm{H} \alpha$ line impossible. The set of $\mathrm{Fe}_{\mathrm{I}}$ lines was also used to quantify the effect of rotational line broadening. Neglecting additional line broadening effects, we obtained an upper limit of $10 \mathrm{~km} \mathrm{~s}^{-1}$ on $v \sin i$ at a confidence level of $90 \%$.

These findings are compatible with the companion being a K9 star (Cox 2000, p. 388, Table 15.7). Our results are in line with those of Alonso et al. (2008) and Gillon et al. (2010), who found the photometric magnitudes measured in optical (Exodat), near-infrared (2MASS), and infrared (Spitzer) filter bands to be consistent with a late-K or early-M type companion star.

To find the radial velocity of the visual companion, we cross-correlated our five companion spectra with a template spectrum corresponding to our best-fit stellar parameters. The radial velocity was estimated independently in our five companion spectra, and corrected for the wavelength drift visible in the telluric lines. The resulting average radial velocity amounts to $23.9 \pm 0.4 \mathrm{~km} \mathrm{~s}^{-1}$, a value close to CoRoT-2a's radial velocity of $23.245 \pm 0.010 \mathrm{~km} \mathrm{~s}^{-1}$, which we determined accordingly. We note that it is also independent of the details of the chosen spectral model. Given the apparent distance in the sky of $4^{\prime \prime}$ and a distance of about $270 \mathrm{pc}$ the projected distance between CoRoT-2a and the companion amounts to about 1100 AU. Because CoRoT-2a is basically solar-like in mass, Kepler's third law yields a lower bound for the orbital period of $40000 \mathrm{a}$, which gives an orbital velocity of up to $0.9 \mathrm{~km} \mathrm{~s}^{-1}$. Thus, the radial velocity found for the visual companion of CoRoT-2a is compatible with the hypothesis of a gravitationally bound companion.

To check for relative sky motions of CoRoT-2a and 2MASS J19270636+0122577, we inspected the photographic data available from the Digitized Sky Survey. We checked the digitized plates of the Palomar Observatory Sky Surveys from 1951, 1983, and 1991 and the HST Guide Star Catalogue from 1980, but found no indications for a relative transversal
Table 4. Stellar parameters of 2MASS J19270636+0122577.

\begin{tabular}{lcc}
\hline \hline Parameter & Value & Lines \\
\hline$T_{\text {eff }}[\mathrm{K}]$ & {$[3900,4100](95 \%$ cred.) } & TiO 7050-7200 \\
$v \sin i\left[\mathrm{~km} \mathrm{~s}^{-1}\right]$ & $<10(90 \% \mathrm{CL})$ & $137 \mathrm{Fe}$ I lines \\
$\mathrm{RV}\left[\mathrm{km} \mathrm{s}^{-1}\right]$ & $23.9 \pm 0.4$ & \\
\hline
\end{tabular}

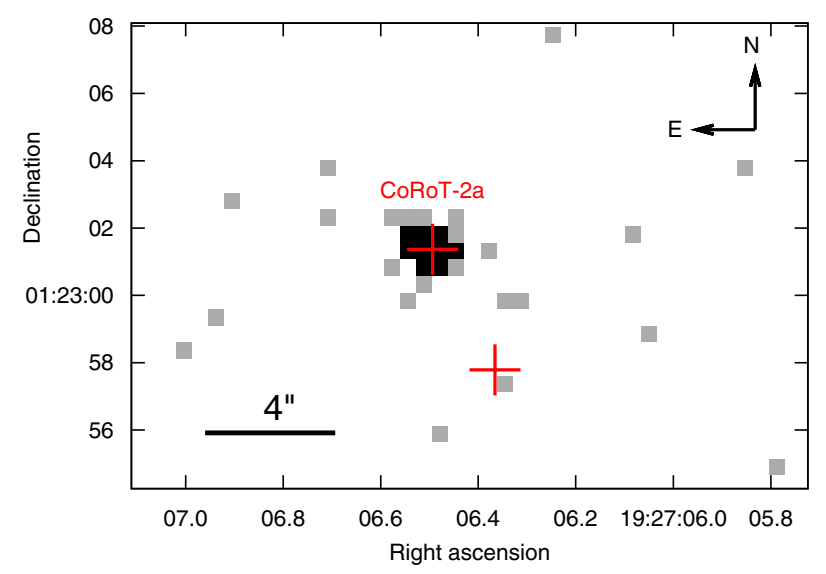

Fig. 7. X-ray image $\left(0.5^{\prime \prime} / \mathrm{bin}\right)$ of the surrounding of CoRoT-2a for energies above $0.3 \mathrm{keV}$. The nominal 2MASS positions of CoRoT-2a and 2MASS J19270636+0122577 are marked with red crosses.

motion. This finding corroborates the hypothesis that 2MASS J19270636+0122577 and CoRoT-2a are gravitationally bound and form a wide binary system.

The parameters derived for the companion are summarized in Table 4.

\section{Analysis of the Chandra X-ray data}

\subsection{Observations}

CoRoT-2a was observed by Chandra using the ACIS-S detector on June 24, 2010 for about 15 ks (Obs.-ID 10989). In the reduction and analysis process, we used the standard software package CIAO in version 4.2. To obtain the best possible timing, the tool axbary was used to apply a barycentric correction to the photon arrival times.

\subsection{Detection and spectral analysis}

In a first step, we screened the X-ray image for photons in the 0.3-4 keV energy band. This step reduces the background contamination and focuses our analysis on the energy band, in which stellar coronal emission is expected to dominate. We show parts of the resulting X-ray image in Fig. 7.

In a second step, we counted all photons within a $2^{\prime \prime}$ radius circular region centered on the nominal position of CoRoT- $2 \mathrm{a}$. In this region, we found 87 photons with an expected background contribution of $\approx 3$ photons, deduced from nearby source-free regions.

Finally, we carried out a spectral analysis of the source photons. Using XSPEC v12.5 Arnaud (1996), we fitted the ACIS spectrum with an absorbed, thermal APEC (e.g., Smith et al. 2001) model. Because the abundances are not well constrained by the fit, we fixed them at their solar values for the rest of the analysis, which is in accordance with our optical estimates (cf. Sect. 2.3). For the depth of the absorbing column, the fit provides a value of $\approx 10^{21} \mathrm{~cm}^{-2}$, which is well compatible 


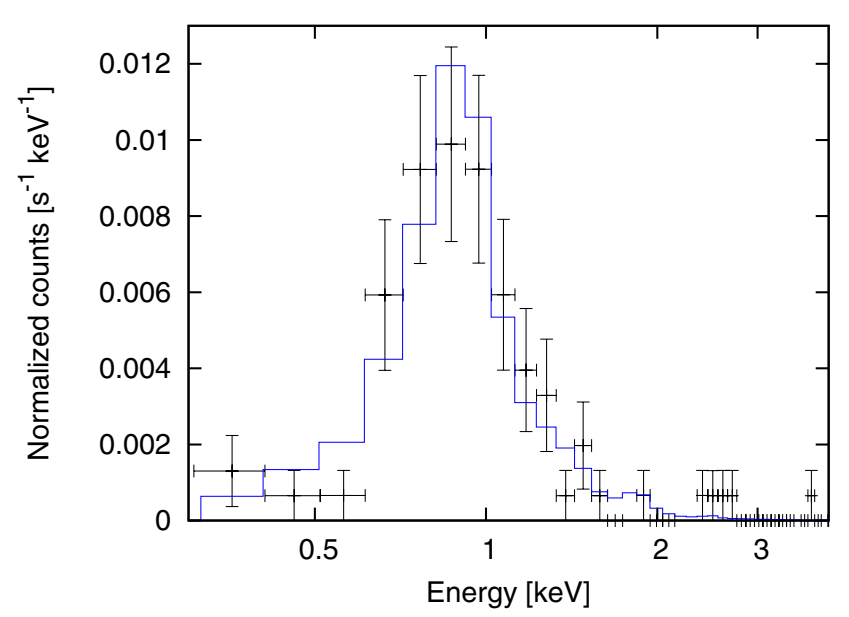

Fig. 8. ACIS-S spectrum of CoRoT-2a with applied spectral model.

Table 5. Spectral-fit results for CoRoT-2a derived from Chandra ACIS$\mathrm{S}$ data.

\begin{tabular}{lc}
\hline \hline Parameter & Value (90\% conf.) \\
\hline$N_{\mathrm{H}}\left[10^{22} \mathrm{~cm}^{-2}\right]$ & $<0.2$ \\
$T[\mathrm{keV}]$ & $0.74(0.57-0.83)$ \\
$T\left[10^{6} \mathrm{~K}\right]$ & $8.6(6.6-9.6)$ \\
$f_{\mathrm{X}}\left[10^{-14} \mathrm{erg} \mathrm{cm}^{-2} \mathrm{~s}^{-1}\right]$ & $2.1(0.8-2.9)$ \\
$L_{\mathrm{X}}($ at $270 \mathrm{pc})\left[10^{29} \mathrm{erg} \mathrm{s}^{-1}\right]$ & $1.9(0.7-2.5)^{a}$ \\
\hline
\end{tabular}

Notes. ${ }^{(a)}$ An error of $\pm 120 \mathrm{pc}$ on the distance estimate translates into a larger conf. interval for $L_{\mathrm{X}}$ of $(0.2-5.2) \times 10^{29} \mathrm{erg} \mathrm{s}^{-1}$.

with a canonical density of 1 particle per $\mathrm{cm}^{3}$ for the interstellar medium and a distance of $\approx 270$ pc (see Sect. 2.8) for CoRoT-2a.

Figure 8 shows the X-ray spectrum and our best-fit model; the fit results are summarized in Table 5. From our best-fit model, we obtain an X-ray luminosity of $L_{\mathrm{X}}=1.9 \times 10^{29} \mathrm{erg} \mathrm{s}^{-1}$ in the $0.3-4 \mathrm{keV}$ band, corresponding to an activity level of $\log _{10} L_{\mathrm{X}} / L_{\mathrm{bol}} \approx-4.2$, which indicates that CoRoT-2a is an active star also by X-ray standards.

\subsection{X-ray light curve and transit}

To investigate the X-ray variability of CoRoT-2a, we constructed background-subtracted light curves with various binnings. A barycentric time-correction was applied to all light curves to obtain time stamps, which can be easily reconciled with planetary ephemerides given in the literature. Figure 9 presents the light curve of CoRoT-2a, which shows no indications of strong shortterm variability like flares, and, therefore, we argue in favor of quiescent emission.

The ephemerides of CoRoT-2b were derived by Alonso et al. (2008) using the CoRoT data. The transit duration is $8208 \mathrm{~s}$, during which the relative flux deficit in the optical reaches $3 \%$. Our Chandra observation completely covers one planetary transit (epoch 651 with respect to the ephemerides from Alonso et al. 2008). The ingress begins $6538 \mathrm{~s}$ after the start of the observation and the egress is finished shortly before the observation ends (cf., Fig. 9).

Our analysis showed that the source count rate, if anything, increased by $17 \%$ during the eclipse. Similarly, the hardness ratio $H R=(H-S) /(H+S)$ with $S=0.3-1 \mathrm{keV}$ and $H=1-4 \mathrm{keV}$ (lower panel in Fig. 9) remains unaffected. On the one hand,

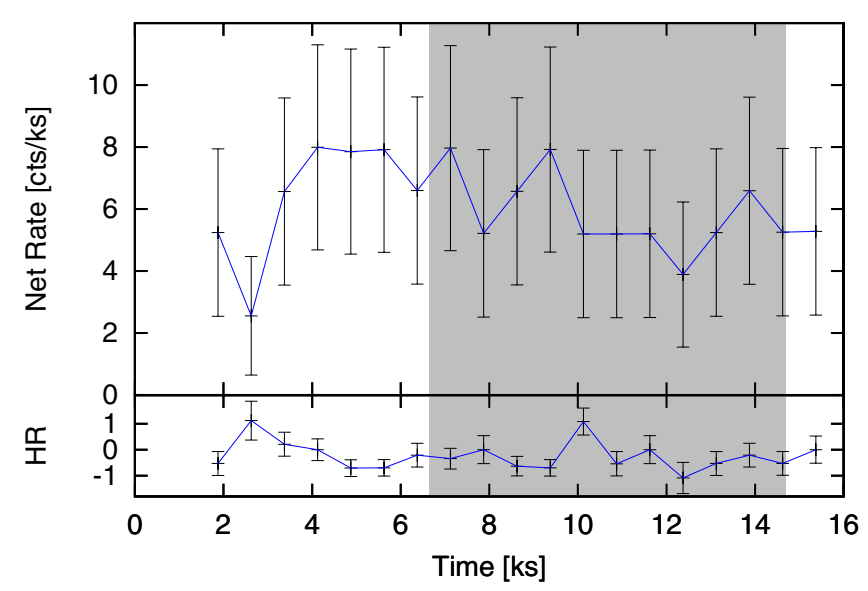

Fig. 9. Light curve of CoRoT-2a in the $0.3-4 \mathrm{keV}$ band and hardness ratio $(H-S) /(H+S)$ for $0.3-1$ and $1-4 \mathrm{keV}$ bands (750 s binning). The shaded area corresponds to the transit in visual wavelengths.

given 82 source counts in $15 \mathrm{ks}$, detecting a $3 \%$ drop in brightness as observed in the optical seems to be out of reach in X-rays. On the other hand, the sources of X-ray emission are believed to be distributed much more inhomogeneously across the stellar surface than those of optical light. We conclude that either the planet did not eclipse a strong concentration of X-ray emitting material in this particular case, the emission is distributed homogeneously, or concentrated at higher latitudes avoided by the planetary disk.

\subsection{The companion in X-rays}

To check whether CoRoT-2a's potentially physical companion, 2MASS J19270636+0122577, is an X-ray source, we collected the photons within a circle of $1^{\prime \prime}$ radius centered on the star's 2MASS position. According to our modeling, this region contains $93 \%$ of the Chandra point spread function (PSF) at $1 \mathrm{keV}$. A single photon with an energy of $1.1 \mathrm{keV}$ was detected in this region. Because $99 \%$ of CoRoT-2a's PSF are confined to a distance of $4^{\prime \prime}$ and less from CoRoT-2a, the detected photon is unlikely to stem from that source. From nearby source-free regions, we estimated the rate of background-counts with energies of $1.1 \pm 0.1 \mathrm{keV}$, where the $0.1 \mathrm{keV}$ range accounts for Chandra's energy resolution, to be $2 \times 10^{-4} \mathrm{cts} \mathrm{s}^{-1}$ within the encircled region centered on the companion.

The detected photon may, consequently, be associated with an X-ray source at the companion's position. To derive an upper limit on the X-ray flux of the companion, we determined the count rate yielding one or less detected photons with a probability of $95 \%$. Consulting Poisson statistics, the limiting count rate amounts to $0.36 \mathrm{cts}$ in $15 \mathrm{ks}$ or $2.4 \times 10^{-5} \mathrm{cts} \mathrm{s}^{-1}$. Assuming that the source has a $1 \mathrm{keV}$ thermal spectrum, a distance of $270 \mathrm{pc}$ (cf. Sect. 2.8), and neglecting absorption, we used WebPIMMS ${ }^{10}$ to convert the count rate into an upper limit of $L_{\mathrm{X}}<9 \times 10^{26} \mathrm{erg} \mathrm{s}^{-1}$ for the companion's X-ray luminosity.

\section{Discussion}

We presented new X-ray and optical data of the active planet host-star CoRoT-2a. Below, we discuss the impact of our findings on our understanding of the CoRoT-2 system.

${ }^{10}$ http://heasarc.gsfc.nasa.gov/Tools/w3pimms.html 


\subsection{CoRoT-2a's atmosphere and activity}

We studied the photosphere of CoRoT-2a applying different techniques of spectroscopic analysis to determine the stellar effective temperature, surface gravity, metallicity, and microturbulence velocity. First, we measured the EW of $238 \mathrm{Fe}$ I and II lines and determined the entire set of parameters by imposing excitation and ionization balance. Second, we redetermined the parameters by directly fitting several sensitive spectral lines and found consistent results, which are, moreover, in line with previously published values (Ammler-von Eiff et al. 2009; Bouchy et al. 2008). With an effective temperature of $5598 \pm 34 \mathrm{~K}$ and a surface gravity of $\log g=4.47 \pm 0,14$, CoRoT-2a is a star of spectral type G6-G7 (Cox 2000, p. 151, Table 7.5) with slightly increased metallicity compared to the Sun. We compared the stellar parameters to those predicted by theoretical isochrone calculations for pre-main and main-sequence stars (Siess et al. 2000). Assuming that CoRoT-2a is close to the zero-age main sequence, these models favor a spectral type of G7 given the observed values of effective temperature and metallicity.

The fact that CoRoT-2a is a highly active star became first obvious in the photometry observed by the CoRoT observatory. The light curve shows pronounced rotational variability caused by active regions that cover a substantial fraction of the stellar photosphere (e.g., Wolter et al. 2009; Czesla et al. 2009; Huber et al. 2009, 2010). This high level of activity, mainly diagnosed by photospheric spots, is expected to be also detectable in chromospheric lines excited by enhanced chromospheric heating.

Indeed, CoRoT-2a shows strong chromospheric emissionline cores in its $\mathrm{Ca}$ II $\mathrm{H}$ and $\mathrm{K}$ lines as well as in the Ca IRT lines. We quantified the strength of the emission by determining the Wilson $\mathrm{S}$-index and a $\log R_{\mathrm{HK}}^{\prime}$ value of $4.458 \pm 0.051$. Our results agree well with those reported by Knutson et al. (2010) and place CoRoT-2a among the most active known planet hoststars. Furthermore, we studied the Ca IRT and derived a value of $0.298 \pm 0.006$ for the $R_{\mathrm{IRT}}$ index, which again demonstrates that CoRoT-2a is a highly active star.

The presence of starspots and strong chromospheric heating suggests that coronal heating is substantial as well. Indeed, our 15 ks Chandra observation yields a clear detection of coronal X-ray emission characterized by a thermal spectrum with a temperature of $1 \mathrm{keV}$. Combining CoRoT-2a's X-ray luminosity of $1.9 \times 10^{29} \mathrm{erg} \mathrm{s}^{-1}$ with its spectral type of G7, we derived an activity level of $\log L_{\mathrm{X}} / L_{\mathrm{bol}} \approx-4.2$, showing that CoRoT-2a is a very active star also by X-ray standards. Although optical studies (Lanza et al. 2009; Huber et al. 2010) suggested a large inhomogeneity in the distribution of active regions, which is very likely also true for the distribution of X-ray emission across the stellar disk, an X-ray transit could neither be detected in the X-ray count rate nor in the hardness ratio. This indicates that either no prominent source of X-ray emission was occulted during this particular transit or that the emission is distributed too homogeneously to cause an X-ray transit detectable with Chandra. In any case, we emphasize that our Chandra snapshot covers no more than $4 \%$ of CoRoT-2a's rotation period and a virtually negligible fraction of the optically observed "beating pattern" (e.g., Alonso et al. 2008) with a period of $\approx 50 \mathrm{~d}$, so that it remains insufficient to obtain a representative picture of CoRoT-2a's corona.

\subsection{The age of CoRoT-2}

One of the key quantities needed to understand the evolution not only of the CoRoT-2 system but of all planetary systems is their age. Based on our analysis, we applied several techniques to estimate the age of CoRoT-2a.

From the EW of the lithium line at $6708 \AA$ in the spectrum of CoRoT-2a, we inferred an age comparable with that of the Pleiades, i.e., $\approx 100$ Ma. Furthermore, we derived a Li abundance of $A_{\mathrm{Li}}=+2.6$ dex, which suggests an age between 100 and $250 \mathrm{Ma}$. Applying the relation provided by Donahue (1998, Eq. (1)), we used the strength of the Ca II $\mathrm{H}$ and $\mathrm{K}$ emissionline cores measured by the $\log R_{\mathrm{HK}}^{\prime}$ index to estimate a "chromospheric age" of $670_{-280}^{+200} \mathrm{Ma}$ for CoRoT-2a. The coronal activity provides another age estimate. Using the relation between $\mathrm{X}$-ray luminosity and age for late-F to early-M dwarfs presented in Sanz-Forcada et al. (2010), we calculated an age of $230_{-40}^{+200} \mathrm{Ma}$. An additional estimate can be obtained from gyrochronology. Using the relation presented by Barnes (2007), we determined an age of $76 \pm 7 \mathrm{Ma}$ for CoRoT-2a. However, Barnes note that gyrochronology tends to underestimate the stellar age if $(B-V)>0.6$, which is true for CoRoT-2a, owing to the sparseness of the open cluster sample used for calibration in case of blue stars.

Guillot \& Havel (2011) modeled the evolution of the star, CoRoT-2a, and its planet simultaneously and found two classes of solutions reproducing the observed properties of the CoRoT-2 system: first, a solution in which CoRoT-2a is a very young star with an age of 30 to $40 \mathrm{Ma}$ and second, a solution with a more evolved main-sequence host-star with an age of 130 to $500 \mathrm{Ma}$. None of the above age indicators, not even the gyrochronological age estimate, favors the solution with a very young host-star, rendering this class of evolutionary scenarios found by Guillot \& Havel (2011) unlikely.

In summary, we conclude that combining our outcomes with published results both observational and theoretical favors an age between 100 and 300 Ma for CoRoT-2a. This suggests that CoRoT-2a is a young main-sequence star that has already left the zero-age main sequence, which for a G7 star of solar mass is situated at an age of $30 \mathrm{Ma}$ (Siess et al. 2000).

\subsection{Distance to CoRoT-2a}

Photometric colors are often used for a rough spectral classification. The magnitudes provided by SIMBAD yield a $B-V$ color index of 0.854 mag for CoRoT-2a. Comparing this value with the color index expected for a G7 star of age 200 Ma with slightly increased metallicity $(Z=0.01)$ (Siess et al. 2000), we calculated a color excess of $E(B-V)=0.15 \mathrm{mag}$. Thus, CoRoT-2a appears redder than expected; we attribute this to interstellar extinction.

Combining the $B-V$ color excess with the relation given by Bohlin et al. (1978)

$\langle N(\mathrm{H} \mathrm{I}+\mathrm{H} \mathrm{II}) / E(B-V)\rangle=5.8 \times 10^{21} \mathrm{~cm}^{-2} \mathrm{mag}^{-1}$,

we obtain a column density of $9 \times 10^{20} \mathrm{~cm}^{-2}$, which is consistent with the upper limit of $2 \times 10^{21} \mathrm{~cm}^{-2}$ derived from our X-ray observations. Converting the EWs of the interstellar Na D absorption lines into a column density of $8 \times 10^{12} \mathrm{~cm}^{-2}$ via the lineratio method (Strömgren 1948), we obtained another consistent estimate of $\approx 10^{21} \mathrm{~cm}^{-2}$ for the hydrogen column density (Ferlet et al. 1985). Assuming a density of one particle per $\mathrm{cm}^{3}$ for the interstellar medium, the hydrogen column density inferred from the color excess directly translates into a distance estimate of $290 \mathrm{pc}$, which is consistent with our previous estimate of $270 \mathrm{pc}$.

According to our spectroscopic analysis, CoRoT-2a can be classified as a G7-type dwarf and, according to the evolutionary 
model, should have an absolute visual brightness of 5.1 mag. Assuming a value of $R=3.1$ (Schultz \& Wiemer 1975) for the ratio of total visual extinction, $A_{V}$, and $E(B-V)$, we derive $A_{V}=0.48 \mathrm{mag}$ for CoRoT-2a. Combining this with the apparent visual brightness of $12.57 \mathrm{mag}$, we derived an extinctioncorrected spectroscopic parallax of $250 \mathrm{pc}$.

In Sect. 2.7 we estimated the distance to the CoRoT-2 system from the Wilson-Bappu width of the $\mathrm{Ca}$ II $\mathrm{H}$ and $\mathrm{K}$ emission line cores and obtained $140_{-40}^{+50}$ to $190_{-50}^{+60} \mathrm{pc}$ depending on the details of the calibration. We further determined the distance from the presence of interstellar $\mathrm{Ca}$ and $\mathrm{Na}$ absorption features in the spectrum and obtained $270 \pm 120$ pc based on the interstellar absorption column (Sect. 2.8). In summary, we argue in favor of a distance of $\approx 270 \mathrm{pc}$ as the most likely value.

\subsection{X-rays eroding CoRoT-2b}

Because CoRoT-2b orbits its host star at a distance of only $0.03 \mathrm{AU}$, it is immersed in an enormous high-energy radiation field. According to CoRoT-2a's X-ray luminosity, the X-ray flux at the distance of CoRoT-2b's orbit amounts to $8.5 \times 10^{4} \mathrm{erg} \mathrm{cm}^{-2} \mathrm{~s}^{-1}$, which is five orders of magnitude larger than the solar X-ray flux received by Earth. This amount of ionizing radiation can have a significant influence on the structure and evolution of the planetary atmosphere. Schneider et al. (1998) found that the extent of the atmospheres of hot Jovian exoplanets can exceed the Roche lobe, leading to evaporation of planetary material by an interaction with the stellar wind. Indeed, extended atmospheres of extrasolar planets were found for HD 209458b (Vidal-Madjar et al. 2003; Linsky et al. 2010) and HD 189733b (Lecavelier Des Etangs et al. 2010).

Sanz-Forcada et al. (2010) analyzed a sample of planetary systems. The authors come to the conclusion that erosion triggered by stellar high-energy illumination has a detectable influence on the observed mass distribution of exoplanets. This gives rise to an "erosion line", below which Sanz-Forcada et al. (2010) find the large majority of the planets in their sample. To estimate the mass loss induced by the X-ray and extreme-UV (EUV) irradiation, we used Eq. (2) from Sanz-Forcada et al. (2011), viz.

$\dot{M}=\frac{3 F_{\mathrm{XUV}}}{4 G \rho}$

where $F_{\text {XUV }}$ is the sum of the stellar X-ray and EUV flux at the planetary orbit, $G$ is the gravitational constant, and $\rho$ is the density of the planet (all in cgs units). Because there are no EUV data of CoRoT-2a available, we use Eq. (3) from Sanz-Forcada et al. (2011), which provides a relation between X-ray and EUV luminosity calibrated with their sample of objects, to obtain an estimate of $4.3 \times 10^{5} \mathrm{erg} \mathrm{cm}^{-2} \mathrm{~s}^{-1}$ for the expected EUV flux at the distance of CoRoT-2b. Substituting the parameters for CoRoT-2b (Alonso et al. 2008, Table 1), we obtained a massloss rate of $4.5 \times 10^{12} \mathrm{~g} \mathrm{~s}^{-1}$ or $7.3 \times 10^{-2} M_{\mathrm{J}} \mathrm{Ga}^{-1}$ for the planet. Given the uncertainties, this value remains a coarse estimate, but places CoRoT-2b clearly above the erosion line, which, according to Sanz-Forcada et al., may be explained by the youth of the system. We note, however, that by using different assumptions for the extent of CoRoT-2b's atmosphere (Sanz-Forcada et al. 2010, Eq. (1)), the mass-loss rate may be increased by up to one order of magnitude. Moreover, the effects leading to planetary mass loss are not yet well understood.

\subsection{The companion - a puzzling genesis}

An important consequence of our analysis is that the CoRoT-2 system may extend far beyond the planetary orbit. CoRoT-2a's visual companion, 2MASS J19270636+0122577, may actually also be a physical companion, forming a wide binary pair with CoRoT-2a.

We obtained and analyzed the first low-SNR UVES spectrum of the companion. Alonso et al. (2008) already noticed that the companion may be a late-K or early-M-type star at about the same distance as CoRoT-2a. We measured the companion's radial velocity and found a value of $23.9 \pm 0.4 \mathrm{~km} \mathrm{~s}^{-1}$, which is close to CoRoT-2a's radial velocity. By modeling the TiO bands present in the spectrum, we determined an effective temperature between 3900 and $4100 \mathrm{~K}$. Wide lines of Ca I were used to infer a surface gravity of $\log g=4.74$. Consulting the evolutionary tracks of Siess et al. (2000) at an estimated age of $200 \mathrm{Ma}$, we find that the companion is likely to be a star of spectral type $\mathrm{K} 9$, which is gravitationally bound to CoRoT-2a. This would make CoRoT-2 one of about 40 known binary systems harboring an exoplanet (Mugrauer \& Neuhäuser 2009).

If this hypothesis withstands further observational tests, it would challenge our understanding of the CoRoT- 2 system, in particular, the age of the system. From the Chandra data we derived an upper limit of $9 \times 10^{26} \mathrm{erg} \mathrm{s}^{-1}$ for the X-ray luminosity of the companion, and the Siess et al. evolutionary tracks suggest an absolute bolometric luminosity of $6.8 \mathrm{mag}$. Combining these numbers, we obtain $\log L_{\mathrm{X}} / L_{\mathrm{bol}}<-5.8$, making the companion a star much less active than CoRoT-2a. From our spectral analysis, we concluded that CoRoT-2a has an age between 100 and $300 \mathrm{Ma}$. Assuming that the companion is physically bound and has the same age as CoRoT-2a, we would expect an X-ray flux significantly higher than observed in our Chandra pointing. From the study of X-ray emission of members of the Pleiades cluster, Micela et al. (1996) find typical X-ray luminosities for $\mathrm{K}$-stars of $\log L_{X}=29.4 \mathrm{erg} \mathrm{s}^{-1}$, which is more than two orders of magnitude above our upper limit for the companion. We therefore conclude that either the companion has never been an active X-ray source, which seems unusual for a young late-type star, or that the activity of the companion has already dropped to a moderate level. Given the upper limit for the X-ray luminosity, the companion may be an evolved K-type star similar to those found in the solar neighborhood (Schmitt et al. 1995). This also agrees with the upper limit on its rotational velocity of $v \sin i<10 \mathrm{~km} \mathrm{~s}^{-1}$; neglecting the unknown inclination, this would be a value typical for K-type stars on the main-sequence (Cox 2000, p. 389, Table 15.8).

We speculate that the CoRoT-2 system, if bound, should be old enough to let the K-star become sufficiently inactive, while the G-star CoRoT-2a remained more active, maybe through an interaction with its close-in planet. This hypothesis is backed by the recent results presented by Brown et al. (2011), who reported on a discrepancy between different age estimations of the host stars of the planetary systems WASP-18 and WASP-19. Both stars harbor a close-in hot Jupiter and appear to be older than attested by their gyrochronological age. Brown et al. (2011) suggested that an inward migration of the hot Jupiters has caused a spin-up of their host stars via tidal interaction. Alternatively, or even additionally, interactions between the planetary and stellar magnetic fields may have reduced the stellar angular-momentum loss as proposed by Lanza (2010). The CoRoT-2 system is among the planetary systems with the shortest orbital periods and should, therefore, be susceptible to these effects. 


\subsection{On the dynamics including the companion}

A gravitationally bound stellar companion influences the dynamics of the CoRoT-2 system. In particular, it slightly disturbs the planetary orbit. Indeed, Gillon et al. (2010) find a temporal offset of the secondary eclipse, which can be attributed to a slight orbit eccentricity or another interacting body. Because transit timing variations larger than $10 \mathrm{~s}$ are excluded by the CoRoT light curve (Alonso et al. 2009), Gillon et al. conclude that the planetary orbit has an eccentricity of $\sim 0.014$. Given the present eccentricity of the orbit, the anomalous radius of the planet can be explained by evolutionary scenarios if the models include a third planetary body in the system. Guillot \& Havel (2011) propose two possible scenarios, which would result in a relatively recent $(\sim 20 \mathrm{Ma})$ start of the circularization process of the orbit. One requires a planetary encounter and the other is based on the Kozai interaction with a distant body. Our findings clearly favor the latter scenario.

\section{Conclusion}

The CoRoT-2 system may be a key to a more profound understanding of the early evolution of planetary systems. We studied new optical and X-ray data. Our analysis showed that magnetic activity can be traced through all layers of the stellar atmosphere from the photosphere to the corona and provided new evidence, helping to answer questions about the age, distance, and evolution of the system. A detailed analysis of several age indicators showed that an age between 100 and $300 \mathrm{Ma}$ is most likely. Furthermore, we were able to provide an estimate of $270 \mathrm{pc}$ for the distance of CoRoT-2a, but with a large uncertainty.

Beyond answering questions, our analysis also raised new problems. Most notably, the true nature of 2MASS J19270636+0122577, the optical and potentially physical stellar companion of CoRoT-2a, remains doubtful. The apparent presence of a gravitationally bound and, therefore, most likely coeval K-type stellar companion, which, nonetheless, shows no detectable activity, would challenge our picture of the CoRoT-2 system. Either the companion is old enough to have already become inactive, or CoRoT-2a appears to be younger than it actually is. A third body would have a substantial impact on the evolutionary dynamics of the whole system. It may account for the eccentricity of the planetary orbit and may even be responsible for the observed anomalously large radius of CoRoT-2b.

Acknowledgements. This work has made use of observational data obtained with UVES at the ESO Very Large Telescope, Paranal, Chile, and the Chandra X-ray Observatory. We acknowledge use of the VALD and NIST atomic line databases, DSS, and SIMBAD. We are indebted to Nikolai Piskunov (University of Uppsala) for making SME available to us. We are thankful to Eric J. Bubar (University of Rochester) for providing us with his MSPAWN and PYSPEC routines. We are grateful to Richard O. Gray (Appalachian State University) for making his SPECTRUM line synthesis code publicly available. We thank Matthias Ammler-von Eiff (Georg-August-Universität Göttingen) for sharing his insight with us. S.S. acknowledges support from the DLR under grant 50OR0703. S.C. and U.W. acknowledge support from the DLR under grant 50OR0105. H.M.M. and K.F.H. are supported in the framework of the DFGfunded Research Training Group "Extrasolar Planets and their Host Stars" (DFG 1351/1).

\section{References}

Alonso, R., Auvergne, M., Baglin, A., et al. 2008, A\&A, 482, L21

Alonso, R., Aigrain, S., Pont, F., Mazeh, T., \& The CoRoT Exoplanet Science Team 2009, in IAU Symp., 253, 91

Alonso, R., Deeg, H. J., Kabath, P., \& Rabus, M. 2010, AJ, 139, 1481
Ammler-von Eiff, M., Santos, N. C., Sousa, S. G., et al. 2009, A\&A, 507, 523 Andretta, V., Busà, I., Gomez, M. T., \& Terranegra, L. 2005, A\&A, 430, 669 Arnaud, K. A. 1996, in Astronomical Data Analysis Software and Systems V, ed. G. H. Jacoby, \& J. Barnes, ASP Conf. Ser., 101, 17

Ayres, T. R. 1979, ApJ, 228, 509

Baliunas, S. L., Donahue, R. A., Soon, W., \& Henry, G. W. 1998, in Cool Stars, Stellar Systems, and the Sun, ed. R. A. Donahue, \& J. A. Bookbinder, ASP Conf. Ser., 154, 153

Barnes, S. A. 2007, ApJ, 669, 1167

Bohlin, R. C., Savage, B. D., \& Drake, J. F. 1978, ApJ, 224, 132

Bouchy, F., Queloz, D., Deleuil, M., et al. 2008, A\&A, 482, L25

Brown, D. J. A., Collier Cameron, A., Hall, C., Hebb, L., \& Smalley, B. 2011 MNRAS, in press [arXiv: 1103.3599]

Bruntt, H., Deleuil, M., Fridlund, M., et al. 2010, A\&A, 519, A51

Bubar, E. J., \& King, J. R. 2010, AJ, 140, 293

Busà, I., Aznar Cuadrado, R., Terranegra, L., Andretta, V., \& Gomez, M. T. 2007, A\&A, 466, 1089

Cox, A. N. 2000, Allen's astrophysical quantities, ed. A. N. Cox,

Cox, D. P., \& Reynolds, R. J. 1987, ARA\&A, 25, 303

Czesla, S., Huber, K. F., Wolter, U., Schröter, S., \& Schmitt, J. H. M. M. 2009, A\&A, 505, 1277

Dekker, H., D’Odorico, S., Kaufer, A., Delabre, B., \& Kotzlowski, H. 2000, in SPIE 4008, ed. M. Iye, \& A. F. Moorwood, 534

Deming, D., Knutson, H., Agol, E., et al. 2011, ApJ, 726, 95

Donahue, R. A. 1998, in Cool Stars, Stellar Systems, and the Sun, ed. R. A. Donahue, \& J. A. Bookbinder, ASP Conf. Ser., 154, 1235

Ferlet, R., Vidal-Madjar, A., \& Gry, C. 1985, ApJ, 298, 838

Fortney, J. J., Lodders, K., Marley, M. S., \& Freedman, R. S. 2008, ApJ, 678, 1419

Fuhrmann, K. 1998, A\&A, 330, 626

Fuhrmann, K. 2004, Astron. Nachr., 325, 3

Gillon, M., Lanotte, A. A., Barman, T., et al. 2010, A\&A, 511, A3

Gray, R. O., \& Corbally, C. J. 1994, AJ, 107, 742

Guillot, T., \& Havel, M. 2011, A\&A, 527, A20

Huber, K. F., Czesla, S., Wolter, U., \& Schmitt, J. H. M. M. 2009, A\&A, 508, 901

Huber, K. F., Czesla, S., Wolter, U., \& Schmitt, J. H. M. M. 2010, A\&A, 514 A39

King, J. R. 1993, AJ, 105, 1087

Knutson, H. A., Howard, A. W., \& Isaacson, H. 2010, ApJ, 720, 1569

König, B., Guenther, E. W., Woitas, J., \& Hatzes, A. P. 2005, A\&A, 435, 215

Kurucz, R. L. 1993, SYNTHE spectrum synthesis programs and line data, ed. R. L. Kurucz

Lallement, R., \& Bertin, P. 1992, A\&A, 266, 479

Lanza, A. F. 2010, A\&A, 512, A77

Lanza, A. F., Pagano, I., Leto, G., et al. 2009, A\&A, 493, 193

Lecavelier Des Etangs, A., Ehrenreich, D., Vidal-Madjar, A., et al. 2010, A\&A, 514, A72

Linsky, J. L. 1980, ARA\&A, 18, 439

Linsky, J. L., Yang, H., France, K., et al. 2010, ApJ, 717, 1291

Megier, A., Strobel, A., Bondar, A., et al. 2005, ApJ, 634, 451

Megier, A., Strobel, A., Galazutdinov, G. A., \& Krełowski, J. 2009, A\&A, 507, 833

Melo, C., Santos, N. C., Pont, F., et al. 2006, A\&A, 460, 251

Micela, G., Sciortino, S., Kashyap, V., Harnden, Jr., F. R., \& Rosner, R. 1996 ApJS, 102, 75

Milone, A., \& Barbuy, B. 1994, A\&AS, 108, 449

Montes, D., Fernandez-Figueroa, M. J., de Castro, E., \& Sanz-Forcada, J. 1997, A\&AS, 125, 263

Mugrauer, M., \& Neuhäuser, R. 2009, A\&A, 494, 373

Noyes, R. W., Weiss, N. O., \& Vaughan, A. H. 1984, ApJ, 287, 769

Pace, G., Pasquini, L., \& Ortolani, S. 2003, A\&A, 401, 997

Pinsonneault, M. H. 1994, in Cool Stars, Stellar Systems, and the Sun, ed. J.-P. Caillault, ASP Conf. Ser., 64, 254

Piskunov, N. E., \& Valenti, J. A. 2002, A\&A, 385, 1095

Piskunov, N. E., Kupka, F., Ryabchikova, T. A., Weiss, W. W., \& Jeffery, C. S. 1995, A\&AS, 112, 525

Plez, B. 1998, A\&A, 337, 495

Reimers, D. 1977, A\&A, 57, 395

Rutten, R. G. M. 1984, A\&A, 130, 353

Sanz-Forcada, J., Ribas, I., Micela, G., et al. 2010, A\&A, 511, L8

Sanz-Forcada, J., Micela, G., Ribas, I., et al. 2011, A\&A, in press [arXiv: 1105.0550$]$

Schmitt, J. H. M. M., Fleming, T. A., \& Giampapa, M. S. 1995, ApJ, 450, 392

Schneider, J., Rauer, H., Lasota, J. P., Bonazzola, S., \& Chassefiere, E. 1998 in Brown Dwarfs and Extrasolar Planets, ed. R. Rebolo, E. L. Martin, \& M. R. Zapatero Osorio, ASP Conf. Ser., 134, 241

Schultz, G. V., \& Wiemer, W. 1975, A\&A, 43, 133 
Sestito, P., \& Randich, S. 2005, A\&A, 442, 615

Sfeir, D. M., Lallement, R., Crifo, F., \& Welsh, B. Y. 1999, A\&A, 346, 785

Siess, L., Dufour, E., \& Forestini, M. 2000, A\&A, 358, 593

Silva-Valio, A., Lanza, A. F., Alonso, R., \& Barge, P. 2010, A\&A, 510, A25

Smith, R. K., Brickhouse, N. S., Liedahl, D. A., \& Raymond, J. C. 2001, ApJ,

556, L91

Sneden, C. A. 1973, Ph.D. thesis, University of Texas at Austin

Snellen, I. A. G., de Mooij, E. J. W., \& Burrows, A. 2010, A\&A, 513, A76

Soderblom, D. R., Oey, M. S., Johnson, D. R. H., \& Stone, R. P. S. 1990, AJ, 99, 595

Soderblom, D. R., Fedele, S. B., Jones, B. F., Stauffer, J. R., \& Prosser, C. F. 1993a, AJ, 106, 1080

Soderblom, D. R., Jones, B. F., Balachandran, S., et al. 1993b, AJ, 106, 1059

Soderblom, D. R., Pilachowski, C. A., Fedele, S. B., \& Jones, B. F. 1993c, AJ, 105,2299
Soderblom, D. R., King, J. R., Siess, L., Jones, B. F., \& Fischer, D. 1999, AJ, 118,1301

Sousa, S. G., Santos, N. C., Israelian, G., Mayor, M., \& Monteiro, M. J. P. F. G. 2007, A\&A, 469, 783

Sousa, S. G., Santos, N. C., Mayor, M., et al. 2008, A\&A, 487, 373

Sousa, S. G., Alapini, A., Israelian, G., \& Santos, N. C. 2010, A\&A, 512, A13

Strömgren, B. 1948, ApJ, 108, 242

Valenti, J. A., \& Fischer, D. A. 2005, ApJS, 159, 141

Valenti, J. A., \& Piskunov, N. 1996, A\&AS, 118, 595

Vaughan, A. H., Preston, G. W., \& Wilson, O. C. 1978, PASP, 90, 267

Vidal-Madjar, A., Lecavelier des Etangs, A., Désert, J., et al. 2003, Nature, 422, 143

Welsh, B. Y., Lallement, R., Vergely, J., \& Raimond, S. 2010, A\&A, 510, A54

Wilson, O. C., \& Bappu, M. K. V. 1957, ApJ, 125, 661

Wolter, U., Schmitt, J. H. M. M., Huber, K. F., et al. 2009, A\&A, 504, 561 